\title{
Exact solution of the Percus-Yevick integral equation for fluid mixtures of hard hyperspheres
}

\author{
René D. Rohrmann* \\ Instituto de Ciencias Astronómicas, de la Tierra y del Espacio (ICATE-CONICET), Avenida España 1512 Sur, 5400 San Juan, Argentina
}

\author{
Andrés Santos ${ }^{\dagger}$ \\ Departamento de Física, Universidad de Extremadura, E-06071 Badajoz, Spain
}

(Received 11 August 2011; published 24 October 2011)

\begin{abstract}
Structural and thermodynamic properties of multicomponent hard-sphere fluids at odd dimensions have recently been derived in the framework of the rational function approximation (RFA) [Rohrmann and Santos, Phys. Rev. E 83, 011201 (2011)]. It is demonstrated here that the RFA technique yields the exact solution of the Percus-Yevick (PY) closure to the Ornstein-Zernike (OZ) equation for binary mixtures at arbitrary odd dimensions. The proof relies mainly on the Fourier transforms $\widehat{c}_{i j}(k)$ of the direct correlation functions defined by the OZ relation. From the analysis of the poles of $\widehat{c}_{i j}(k)$ we show that the direct correlation functions evaluated by the RFA method vanish outside the hard core, as required by the PY theory.
\end{abstract}

DOI: 10.1103/PhysRevE.84.041203 PACS number(s): 61.20.Gy, 61.20.Ne, 05.20.Jj, 51.30.+i

\section{INTRODUCTION}

The understanding and interpretation of thermodynamic properties and the structure of real dense gases and liquids rely mostly on the use of models of fluids and the application of approximate theories [1]. One of the most successful and widely used approximate methods is the Percus-Yevick (PY) theory. It is an integral equation theory based on the Ornstein-Zernike (OZ) equation [2] coupled with the PY closure [3]. Systems of rigid nonattracting hard spheres (HS), as well as their modified forms (e.g., square-well and sticky particles), represent useful fluid models for the study of liquids and colloidal systems [4]. In this context, it is noteworthy that exact solutions of the PY integral equation were early obtained for pure [5,6] and multicomponent [7] HS fluids.

Recently, we have shown [8,9] that the thermodynamic and structural properties of single and (additive) multicomponent fluids of hard hyperspheres at odd dimensions can be studied by means of an analytical technique, the so-called rational function approximation (RFA). This method is formulated in terms of the Laplace transform of a polynomial times the radial distribution function and leads directly to a system of algebraic equations that have analytical solution at low spatial dimensionality (odd $d \leqslant 7$ for pure fluids and odd $d \leqslant 3$ for mixtures) and must be solved numerically at higher odd dimensions.

In the single-component case, it was shown that, in its simplest formulation, the RFA method recovers the known solution of the PY closure to the OZ equation [8]. In the multicomponent case, the equivalence between the solutions of the two approximations has been shown for binary mixtures in three dimensions [10] and five dimensions [9]. Although compelling arguments were presented in Ref. [9] in favor of the extension of the equivalence to general odd $d$, a rigorous proof was not attempted. Therefore, whether or not the PY integral equation is exactly solvable for mixtures of additive hard hyperspheres with arbitrary $d=$ odd (as well as the

*rohr@icate-conicet.gob.ar http://icate-conicet.gob.ar/rohrmann. †andres@unex.es http://www.unex.es/eweb/fisteor/andres. structure of the solution in the affirmative case) remained, to the best of our knowledge, an open problem.

The purpose of the present paper is to give a formal proof that the PY and RFA solutions are indeed equivalent for binary mixtures in arbitrary odd dimensions. The proof is based on the analysis of the Fourier transform $\widehat{c}_{i j}(k)$ of the direct correlation function $c_{i j}(r)$, which is defined by the OZ equation and is a key quantity to be determined by both approximations. The equivalence between RFA and PY theories is ensured by first deriving the functional structure of $\widehat{c}_{i j}(k)$ as given by the RFA. The direct correlation function $c_{i j}(r)$ is then calculated by the theory of residues, showing that it is strictly short-ranged, as expressed by the PY closure.

The organization of the paper is as follows. Section II provides a description of the fluid structure equations and basic definitions of the two approximations. Section III particularizes the RFA scheme to binary mixtures of HS at odd dimensionalities. Section IV deals with the analysis of the direct correlation function and its short-range behavior as derived from the RFA theory. We close the paper in Sec. V with some concluding remarks.

\section{FORMULATION}

\section{A. Basic quantities}

Let us consider an $\mathcal{N}$-component fluid mixture of additive $d$-dimensional HS. Let $\left\{x_{i} ; i=1, \ldots, \mathcal{N}\right\}$ be the set of mole fractions, $\left\{\sigma_{i} ; i=1, \ldots, \mathcal{N}\right\}$ be the set of diameters, and $\rho$ be the total number density.

The radial distribution function $g_{i j}(r)$, the total correlation function $h_{i j}(r)$, and the direct correlation function $c_{i j}(r)$ corresponding to particle pairs of species $i$ and $j$ are the primary linkage between thermodynamic and structural properties and particle interactions of multicomponent fluid mixtures. They are not independent quantities. In particular, $h_{i j}(r)=g_{i j}(r)-1$, while $c_{i j}(r)$ is defined in terms of $h_{i j}(r)$ by the OZ equation, which is conveniently written in Fourier space and in matrix form as

$$
\widetilde{\mathrm{C}}(k)=\mathrm{I}-[\mathrm{I}+\tilde{\mathrm{h}}(k)]^{-1}=\tilde{\mathrm{h}}(k) \cdot[\mathrm{I}+\widetilde{\mathrm{h}}(k)]^{-1} .
$$


Here I is the $\mathcal{N} \times \mathcal{N}$ unit matrix and $\widetilde{\mathrm{c}}(k)$ and $\widetilde{\mathrm{h}}(k)$ are $\mathcal{N} \times$ $\mathcal{N}$ matrices with elements $\rho \sqrt{x_{i} x_{j} c_{i j}}(k)$ and $\rho \sqrt{x_{i} x_{j}} \widehat{h}_{i j}(k)$, respectively, where the hat symbol $($ ) denotes the Fourier transform.

The Fourier transform of the total correlation function can be evaluated from

$$
\widehat{h}_{i j}(k)=v\left[\frac{G_{i j}(s)-G_{i j}(-s)}{s^{d-2}}\right]_{s=i k}, \quad v \equiv(-2 \pi)^{(d-1) / 2},
$$

where $i$ is the imaginary unit and $G_{i j}(s)$ is a Laplace functional of the radial distribution function defined by [9]

$$
G_{i j}(s)=\int_{0}^{\infty} d r \operatorname{rg}_{i j}(r) \theta_{n}(s r) e^{-s r},
$$

with

$$
\theta_{n}(t)=\sum_{\ell=0}^{n} \omega_{n, \ell} t^{\ell}, \quad \omega_{n, \ell} \equiv \frac{(2 n-\ell) !}{2^{n-\ell}(n-\ell) ! \ell !},
$$

being the reverse Bessel polynomial of degree $n=(d-3) / 2$. The function $G_{i j}(s)$ is a fundamental quantity in the sense that it contains all the information about the thermodynamic properties of the fluid and its knowledge gives structural information equivalent to pair radial distributions, pair correlation functions, and structure factors.

Since the HS particles are not allowed to penetrate each other, the radial distribution function vanishes inside the core, i.e.,

$$
g_{i j}(r)=1+h_{i j}(r)=0, \quad r<\sigma_{i j},
$$

where $\sigma_{i j}=\frac{1}{2}\left(\sigma_{i}+\sigma_{j}\right)$ is the contact distance for hyperspheres of species $i$ and $j$. In addition, the exact Laplace functionals $G_{i j}(s)$ for $d$-dimensional HS satisfy the following asymptotic relations [9].

(i) Long wave-number limit:

$$
\lim _{s \rightarrow \infty} s^{(5-d) / 2} e^{\sigma_{i j} s} G_{i j}(s)=\sigma_{i j}^{(d-1) / 2} g_{i j}\left(\sigma_{i j}^{+}\right) .
$$

(ii) Short wave-number expansion:

$$
G_{i j}(s)=\frac{(d-2) ! !}{s^{2}}+\sum_{m=0}^{\infty} \alpha_{n, m} H_{i j, m+1} s^{m},
$$

where

$$
\begin{gathered}
\alpha_{n, m}=\sum_{\ell=0}^{\min (n, m)} \frac{(-1)^{m-\ell}}{(m-\ell) !} \omega_{n, \ell}, \\
H_{i j, m}=\int_{0}^{\infty} d r h_{i j}(r) r^{m} .
\end{gathered}
$$

(iii) Low-density expansion:

$$
\begin{aligned}
G_{i j}(s)= & \frac{\theta_{n+1}\left(\sigma_{i j} s\right) e^{-\sigma_{i j} s}}{s^{2}}+\rho \sum_{\ell=1}^{\mathcal{N}} x_{\ell} \int_{\sigma_{i j}}^{\infty} d r r \theta_{n}(s r) \\
& \times \Omega_{\sigma_{i \ell}, \sigma_{j \ell}}(r) e^{-s r}+\mathcal{O}\left(\rho^{2}\right)
\end{aligned}
$$

where $\Omega_{a, b}(r)$ is the intersection volume of two hyperspheres of radii $a$ and $b$ whose centers are separated by a distance $r \leqslant a+b$.
Conditions (i)-(iii) are consequences of the hard-core interaction and therefore are directly related to the stepfunction structure of $g_{i j}(r)$ [Eq. (2.5)]. Because $\alpha_{n, 2 q+1}=0$ for $q=0, \ldots, n-1$ [8], condition (ii) guarantees through (2.2) that the correlation functions remain bounded at the limit of zero wave-number,

$$
\widehat{h}_{i j}(0)<\infty,
$$

and this in turn assures that the isothermal compressibility of the fluid takes finite values [9].

\section{B. The PY approximation}

The PY approximation to a classical fluid is obtained from the $\mathrm{OZ}$ equation (2.1) supplemented by a particular closure relation between $g_{i j}(r)$ and $c_{i j}(r)$. For hard-hypersphere systems, the PY closure reduces to Eq. (2.5) and the assumption that the direct correlation function is short ranged, namely,

$$
c_{i j}(r)=0, \quad r>\sigma_{i j} .
$$

Therefore, if an approximate radial distribution function $g_{i j}(r)$ satisfies the hard-core condition (2.5) and its associated direct correlation function, as obtained from Eq. (2.1), verifies the condition (2.12), it is necessarily a solution to the PY theory.

\section{The RFA}

In contrast to the PY approach, the RFA method is based on the Laplace functionals $G_{i j}(s)$ rather than on the $\mathrm{OZ}$ equation. Specifically, the RFA provides an analytical representation of $G_{i j}(s)$ that complies with the consistency conditions (i)-(iii) [9]. This analytical approximation reads [11]

$$
G_{i j}(s)=s^{d-2} e^{-\sigma_{i j} s}\left[\mathrm{~L}(s) \cdot \mathrm{B}^{-1}(s)\right]_{i j},
$$

where $\mathrm{L}(s)$ and $\mathrm{B}(s)$ are $\mathcal{N} \times \mathcal{N}$ matrices with elements

$$
\begin{gathered}
L_{i j}(s)=\sum_{m=0}^{n+1} L_{i j}^{(m)} s^{m}, \\
B_{i j}(s)=s^{d} \delta_{i j}-v \rho_{i} e^{-\sigma_{i} s} L_{i j}(s)+P_{i j}(s) .
\end{gathered}
$$

In Eq. (2.15), $\delta_{i j}$ is the Kronecker delta symbol, $\rho_{i}=\rho x_{i}$ is the partial number density of species $i$, and

$$
P_{i j}(s)=v \rho_{i} \sum_{m=0}^{n+1}\left[\sum_{\ell=0}^{d-m} \frac{\left(-\sigma_{i} s\right)^{\ell}}{\ell !}\right] L_{i j}^{(m)} s^{m} .
$$

Note that the matrices $\mathrm{L}(s)$ and $\mathrm{P}(s)$ have polynomial dependencies on $s$ of degrees $n+1=(d-1) / 2$ and $2 n+3=d$, respectively.

The coefficients $L_{i j}^{(m)}, m=0,1, \ldots, n+1=(d-1) / 2$ may depend on the fluid density, the particle diameters, and the component abundances, but they are independent of $s$. Those coefficients are determined from a set of $n+2$ algebraic matrix equations stemming from the requirement of condition (2.7). The reader is urged to consult Ref. [9] for further details.

Note that Eq. (2.13) can be rewritten as

$$
G_{i j}(s)=s^{d-2} e^{-\sigma_{i j} s} \frac{F_{i j}(s)}{\beta(s)},
$$


where

$$
\mathrm{F}(s) \equiv \mathrm{L}(s) \cdot \operatorname{adj}[\mathrm{B}(s)], \quad \beta(s) \equiv|\mathrm{B}(s)| .
$$

Here $\operatorname{adj}(A)$ and $|A|$ refer to the adjoint and the determinant, respectively, of a matrix $A$. Once the coefficients $L_{i j}^{(m)}$ are obtained as functions of $\rho,\left\{\sigma_{i}\right\}$, and $\left\{x_{i}\right\}$, the functionals $G_{i j}(s)$ are fully determined from Eqs. (2.13) or (2.17). Then, the total correlation functions in $k$ space are obtained from Eq. (2.2), which can be rewritten using (2.17) as

$$
\widehat{h}_{i j}(k)=v \frac{R_{i j}(i k)}{\beta(i k) \beta(-i k)},
$$

where

$$
R_{i j}(s) \equiv F_{i j}(s) \beta(-s) e^{-\sigma_{i j} s}+F_{i j}(-s) \beta(s) e^{\sigma_{i j} s} .
$$

Finally, to obtain the direct correlation functions in the configuration space, one makes use of Eq. (2.1) and the inverse Fourier transform [9],

$$
c_{i j}(r)=\frac{(2 \pi)^{-(d+1) / 2}}{r^{d-2}} i \int_{-\infty}^{\infty} d k k \widehat{c}_{i j}(k) \theta_{n}(i k r) e^{-i k r} .
$$

It is worth noting that, as shown in Ref. [9], the Laplace functionals obtained from (2.13) satisfy the asymptotic behaviors given by Eqs. (2.6)-(2.10). In particular, the physical hard-core requirement given by Eq. (2.5) is verified. Therefore, in order to prove the equivalence between the PY and RFA approaches it will be sufficient to show that the direct correlation functions calculated with Eqs. (2.1), (2.19), and (2.21) are short ranged, as required by Eq. (2.12).

Before closing this section, let us derive some properties for small $s$ that will be useful in Sec. IV. By expanding the exponential in Eq. (2.15) and inserting Eq. (2.16), one gets

$B_{i j}(s)=s^{d} \delta_{i j}-v \rho_{i} \sum_{m=0}^{n+1}\left[\sum_{\ell=d-m+1}^{\infty} \frac{\left(-\sigma_{i} s\right)^{\ell}}{\ell !}\right] L_{i j}^{(m)} s^{m}$.

Therefore, $B_{i j}(s)=s^{d}\left[\delta_{i j}+\mathcal{O}(s)\right]$, so that the determinant is

$$
\beta(s)=s^{\mathcal{N} d}[1+\mathcal{O}(s)] .
$$

Next, from Eqs. (2.7) and (2.17) we get

$$
e^{-\sigma_{i j} s} F_{i j}(s)=(d-2) ! ! \frac{\beta(s)}{s^{d}}\left[1+s^{2} \mathcal{F}_{i j}\left(s^{2}\right)+\mathcal{O}\left(s^{d}\right)\right],
$$

where $\mathcal{F}_{i j}\left(s^{2}\right)$ is a polynomial of degree $n=(d-3) / 2$ in $s^{2}$ whose explicit form will not be needed here. In fact, the polynomial $\mathcal{F}_{i j}\left(s^{2}\right)$ cancels in Eq. (2.20), resulting in $R_{i j}(s)=$ $\mathcal{O}\left(s^{2 \mathcal{N} d}\right)$. From Eqs. (2.23) and (2.24) one obtains

$$
F_{i j}(s)=(d-2) ! ! s^{(\mathcal{N}-1) d}[1+\mathcal{O}(s)] .
$$

Particularizing to the binary case $(\mathcal{N}=2)$ and taking the determinant on both sides of Eq. (2.24), we get

$$
e^{-2 \sigma_{12} s}|\mathrm{~F}(s)|=\left[\frac{\beta(s)}{s^{d}}\right]^{2} s^{2}\left[\mathcal{F}\left(s^{2}\right)+\mathcal{O}\left(s^{d-2}\right)\right],
$$

where $\mathcal{F}\left(s^{2}\right)$ is a polynomial of degree $n=(d-3) / 2$ in $s^{2}$. Combination of Eqs. (2.24) and (2.26) yields

$$
e^{\left(2 \sigma_{12}-\sigma_{i j}\right) s}|\mathrm{~L}(-s)| F_{i j}(s)=\frac{\beta(-s) \beta(s)}{s^{3 d-2}}\left[\mathcal{L}_{i j}\left(s^{2}\right)+\mathcal{O}\left(s^{d-2}\right)\right],
$$

where $\mathcal{L}_{i j}\left(s^{2}\right)$ is again a polynomial of degree $n=(d-3) / 2$ and use has been made of the property

$$
|\mathrm{F}(s)|=\beta(s)|\mathrm{L}(s)|,
$$

which follows from Eq. (2.18). Finally, Eq. (2.27) implies

$$
\begin{aligned}
& e^{\left(2 \sigma_{12}-\sigma_{i j}\right) s}|\mathrm{~L}(-s)| F_{i j}(s)+e^{-\left(2 \sigma_{12}-\sigma_{i j}\right) s}|\mathrm{~L}(s)| F_{i j}(-s) \\
& \quad=\frac{\beta(-s) \beta(s)}{s^{3 d-2}} \mathcal{O}\left(s^{d-2}\right)=\mathcal{O}\left(s^{2 d}\right),
\end{aligned}
$$

where in the last equality we have taken into account Eq. (2.23).

\section{BINARY MIXTURES}

Henceforth, we consider an additive system of two components $(\mathcal{N}=2)$. From Eq. (2.1), we have

$$
\widehat{c}_{11}(k)=\frac{\widehat{h}_{11}(k)+\rho_{2}|\widehat{h}(k)|}{D(k)}, \quad \widehat{c}_{12}(k)=\frac{\widehat{h}_{12}(k)}{D(k)},
$$

with

$$
D(k) \equiv 1+\rho_{1} \widehat{h}_{11}(k)+\rho_{2} \widehat{h}_{22}(k)+\rho_{1} \rho_{2}|\widehat{h}(k)| .
$$

The expressions for $\widehat{c}_{22}(k)$ and $\widehat{c}_{21}(k)$ are obtained from those of $\widehat{c}_{11}(k)$ and $\widehat{c}_{12}(k)$ by exchanging the subscripts 1 and 2 .

Equations (3.1) and (3.2) are general and valid for any binary mixture. Now we particularize to the RFA. Using Eq. (2.19), one obtains

$$
\begin{gathered}
D(k)=\frac{\mathcal{D}(i k)}{\beta(i k) \beta(-i k)}, \\
\widehat{c}_{11}(k)=v \frac{R_{11}(i k)+v \rho_{2}|\mathrm{R}(i k)| /[\beta(i k) \beta(-i k)]}{\mathcal{D}(i k)}, \\
\widehat{c}_{12}(k)=v \frac{R_{12}(i k)}{\mathcal{D}(i k)},
\end{gathered}
$$

where

$$
\begin{aligned}
\mathcal{D}(s) \equiv & \beta(s) \beta(-s)+v\left[\rho_{1} R_{11}(s)+\rho_{2} R_{22}(s)\right] \\
& +v^{2} \rho_{1} \rho_{2} \frac{|\mathrm{R}(s)|}{\beta(s) \beta(-s)} .
\end{aligned}
$$

Explicit calculation using Eq. (2.20) gives

$$
\begin{gathered}
\frac{|\mathrm{R}(s)|}{\beta(s) \beta(-s)}=\Phi(s)+\Phi(-s), \\
\mathcal{D}(s)=\beta(s) \beta(-s)+\Lambda(s)+\Lambda(-s),
\end{gathered}
$$

where

$$
\begin{aligned}
\Phi(s) \equiv & |\mathrm{L}(s)| \beta(-s) e^{-\left(\sigma_{1}+\sigma_{2}\right) s}-F_{12}(s) F_{21}(-s) \\
& +F_{11}(s) F_{22}(-s) e^{-\left(\sigma_{1}-\sigma_{2}\right) s} \\
\Lambda(s) \equiv & v \beta(-s)\left[\rho_{1} F_{11}(s) e^{-\sigma_{1} s}+\rho_{2} F_{22}(s) e^{-\sigma_{2} s}\right] \\
& +v^{2} \rho_{1} \rho_{2} \Phi(s) .
\end{aligned}
$$

In Eq. (3.9) we have used Eq. (2.28).

Thus far, we have not used in this section the explicit form of the matrix $\mathrm{B}(s)$, Eq. (2.15). Evaluation of Eq. (2.18) for binary mixtures yields

$$
\mathrm{F}(s)=\left[\begin{array}{lc}
\widetilde{F}_{11}(s)-v \rho_{2}|\mathrm{~L}(s)| e^{-\sigma_{2} s} & \widetilde{F}_{12}(s) \\
\widetilde{F}_{21}(s) & \widetilde{F}_{22}(s)-v \rho_{1}|\mathrm{~L}(s)| e^{-\sigma_{1} s}
\end{array}\right],
$$


where

$$
\begin{aligned}
& \widetilde{F}_{11}(s) \equiv L_{11}(s)\left[s^{d}+P_{22}(s)\right]-L_{12}(s) P_{21}(s), \\
& \widetilde{F}_{12}(s) \equiv L_{12}(s)\left[s^{d}+P_{11}(s)\right]-L_{11}(s) P_{12}(s) .
\end{aligned}
$$

Of course, $\widetilde{F}_{22}(s)$ and $\widetilde{F}_{21}(s)$ are obtained from Eqs. (3.12) and (3.13) by exchanging the subscripts 1 and 2 . In addition, the determinant $\beta(s)$ of the matrix $\mathrm{B}(s)$ can be expressed as

$$
\begin{aligned}
\beta(s)= & \widetilde{\beta}(s)+v^{2} \rho_{1} \rho_{2}|\mathrm{~L}(s)| e^{-\left(\sigma_{1}+\sigma_{2}\right) s} \\
& -v \rho_{1} \widetilde{F}_{11}(s) e^{-\sigma_{1} s}-v \rho_{2} \widetilde{F}_{22}(s) e^{-\sigma_{2} s},
\end{aligned}
$$

with

$$
\widetilde{\beta}(s) \equiv s^{2 d}+s^{d}\left[P_{11}(s)+P_{22}(s)\right]+|\mathrm{P}(s)| .
$$

We can observe that $\widetilde{F}_{i j}(s)$ and $\widetilde{\beta}(s)$ are polynomials in $s$ of degrees $3 n+4=(3 d-1) / 2$ and $2(2 n+3)=2 d$, respectively. In contrast, the functions $F_{11}(s), F_{22}(s), \beta(s)$, $\Phi(s)$, and $\Lambda(s)$ contain exponential terms. The key point, however, is that those exponential terms compensate exactly in the function $\mathcal{D}(s)$. Using Eqs. (3.9), (3.10), (3.11), and (3.14) in Eq. (3.8), one finds, after some algebra,

$$
\begin{aligned}
\mathcal{D}(s)= & \widetilde{\beta}(s) \widetilde{\beta}(-s)+v^{4} \rho_{1}^{2} \rho_{2}^{2}|\mathrm{~L}(s)||\mathrm{L}(-s)| \\
& -v^{2} \sum_{i, j=1}^{2} \rho_{i} \rho_{j} \widetilde{F}_{i j}(s) \widetilde{F}_{j i}(-s) .
\end{aligned}
$$

Thus, $\mathcal{D}(s)$ is an even polynomial of degree $4(2 n+3)=4 d$.

The direct correlation functions in Fourier space are given by Eqs. (3.4) and (3.5). From Eqs. (2.20), (3.7), (3.9), (3.11), and (3.14) it is possible to get

$$
\begin{aligned}
\widehat{c}_{i j}(k)= & \frac{v}{\mathcal{D}(i k)}\left[\widetilde{\mathcal{P}}_{i j}(i k) e^{i \sigma_{i j} k}+\widetilde{\mathcal{P}}_{i j}(-i k) e^{-i \sigma_{i j} k}\right. \\
& \left.+\widetilde{\mathcal{Q}}_{i j}(i k) e^{i\left(\sigma_{i}-\sigma_{j}\right) k / 2}+\widetilde{\mathcal{Q}}_{i j}(-i k) e^{i\left(\sigma_{j}-\sigma_{i}\right) k / 2}\right],
\end{aligned}
$$

where $\widetilde{\mathcal{P}}_{i j}(s)$ and $\widetilde{\mathcal{Q}}_{i j}(s)$ are polynomials of degrees $7 n+10=$ $(7 d-1) / 2$ and $2(3 n+4)=3 d-1$, respectively, given by

$$
\begin{gathered}
\widetilde{\mathcal{P}}_{11}(s)=\widetilde{F}_{11}(-s) \widetilde{\beta}(s)-v^{2} \rho_{2}^{2}|\mathrm{~L}(-s)| \widetilde{F}_{22}(s) \\
\widetilde{\mathcal{P}}_{12}(s)= \\
\widetilde{F}_{12}(-s) \widetilde{\beta}(s)+v^{2} \rho_{1} \rho_{2}|\mathrm{~L}(-s)| \widetilde{F}_{12}(s), \\
\widetilde{\mathcal{Q}}_{11}(s)=-v\left[\rho_{1} \widetilde{F}_{11}(-s) \widetilde{F}_{11}(s)+\rho_{2} \widetilde{F}_{12}(-s) \widetilde{F}_{21}(s)\right] \\
\quad+v^{3} \rho_{1} \rho_{2}^{2}|\mathrm{~L}(s)||\mathrm{L}(-s)|, \\
\widetilde{\mathcal{Q}}_{12}(s)=-v\left[\rho_{1} \widetilde{F}_{11}(-s) \widetilde{F}_{12}(s)+\rho_{2} \widetilde{F}_{12}(-s) \widetilde{F}_{22}(s)\right] .
\end{gathered}
$$

Again, $\widetilde{\mathcal{P}}_{22}(s), \widetilde{\mathcal{P}}_{21}(s), \widetilde{\mathcal{Q}}_{22}(s)$, and $\widetilde{\mathcal{Q}}_{21}(s)$ are obtained by exchanging the subscripts 1 and 2 .

\section{ANALYSIS}

In the derivation of the results in Sec. III we have not needed to use either Eq. (2.16) or the conditions that the coefficients $L_{i j}^{(m)}$ must satisfy [which are summarized by Eq. (2.24)]. In fact, Eq. (3.17) alone is not sufficient to prove the PY condition (2.12).

As said before, the RFA method guarantees that $\widehat{h}_{i j}(k)$ takes finite values at the limit of zero wave-number, Eq. (2.11). Thus, from Eq. (3.2), $D(k)$ remains bounded at $k=0$, and according to Eq. (3.3), we have

$$
\lim _{s \rightarrow 0} \frac{\mathcal{D}(s)}{\beta(s) \beta(-s)}=D(0)<\infty .
$$

Taking into account that, in the binary case $(\mathcal{N}=2), \beta(s)=$ $s^{2 d}$ for small $s$ [see Eq. (2.23)], we have $\mathcal{D}(s)=\mathcal{O}\left(s^{4 d}\right)$. Since, according to Eq. (3.16), $\mathcal{D}(s)$ is a polynomial of degree $4 d$, we conclude that the $\mathcal{D}(s)$ is just a pure power law, i.e.,

$$
\mathcal{D}(s)=D(0) s^{4 d} .
$$

This is the crucial result allowing one to prove Eq. (2.12) from Eq. (3.17). Before proceeding to the proof, let us first simplify Eq. (3.17) a little more.

As shown by Eqs. (3.18)-(3.21), $\widetilde{\mathcal{P}}_{i j}(s)$ and $\widetilde{\mathcal{Q}}_{i j}(s)$ are polynomials of degrees $7 n+10=(7 d-1) / 2$ and $2(3 n+$ 4) $=3 d-1$, respectively. Going back to the quantities $F_{i j}(s)$ and $\beta(s)$, Eqs. (3.18)-(3.21) can be rewritten as

$$
\begin{aligned}
\widetilde{\mathcal{P}}_{i j}(s)= & \widetilde{F}_{i j}(-s) \beta(s)+v F_{i j}(-s) \sum_{k=1}^{2} \rho_{k} e^{-\sigma_{k} s} F_{k k}(s) \\
& +v^{2} \rho_{1} \rho_{2} e^{-\sigma_{i j} s}\left[e^{\left(2 \sigma_{12}-\sigma_{i j}\right) s}|\mathrm{~L}(-s)| F_{i j}(s)\right. \\
& \left.+e^{-\left(2 \sigma_{12}-\sigma_{i j}\right) s}|\mathrm{~L}(s)| F_{i j}(-s)\right], \\
\widetilde{\mathcal{Q}}_{i j}(s)= & -v \sum_{k=1}^{2} \rho_{k} F_{i k}(-s) F_{k j}(s) \\
& -v^{2} \rho_{1} \rho_{2} e^{-\left(\sigma_{i}-\sigma_{j}\right) s / 2}\left[e^{\left(2 \sigma_{12}-\sigma_{i j}\right) s}|\mathrm{~L}(-s)| F_{i j}(s)\right. \\
& \left.+e^{-\left(2 \sigma_{12}-\sigma_{i j}\right) s}|\mathrm{~L}(s)| F_{i j}(-s)\right] .
\end{aligned}
$$

While Eqs. (4.3) and (4.4) conceal the polynomial character of $\widetilde{\mathcal{P}}_{i j}(s)$ and $\widetilde{\mathcal{Q}}_{i j}(s)$, they show, with the help of Eqs. (2.23), (2.25), and (2.29), that

$$
\widetilde{\mathcal{P}}_{i j}(s)=\mathcal{O}\left(s^{2 d}\right), \quad \widetilde{\mathcal{Q}}_{i j}(s)=\mathcal{O}\left(s^{2 d}\right) .
$$

This result, along with Eq. (4.2), allows us to rewrite Eq. (3.17) as

$$
\begin{aligned}
\widehat{c}_{i j}(k)= & \frac{1}{k^{2 d}}\left[\mathcal{P}_{i j}(i k) e^{i \sigma_{i j} k}+\mathcal{P}_{i j}(-i k) e^{-i \sigma_{i j} k}\right. \\
& \left.+\mathcal{Q}_{i j}(i k) e^{i\left(\sigma_{i}-\sigma_{j}\right) k / 2}+\mathcal{Q}_{i j}(-i k) e^{i\left(\sigma_{j}-\sigma_{i}\right) k / 2}\right],
\end{aligned}
$$

where

$$
\mathcal{P}_{i j}(s)=-\frac{v}{D(0)} s^{-2 d} \widetilde{\mathcal{P}}_{i j}(s)
$$

is a polynomial of degree $3 n+4=3(d-1) / 2$ and

$$
\mathcal{Q}_{i j}(s)=-\frac{v}{D(0)} s^{-2 d} \widetilde{\mathcal{Q}}_{i j}(s)
$$

is a polynomial of degree $2(n+1)=d-1$.

Equation (4.6) is the main result of this paper. It provides the functional structure of the Fourier transform $\widehat{c}_{i j}(k)$ in the RFA approach. The direct correlations function in the configuration space $c_{i j}(r)$ can be calculated by application of the residue theorem combining Eqs. (2.21) and (4.6). Since $\widehat{c}_{i j}(0)=$ finite, the integrand in Eq. (2.21) is regular along the real axis, and so we can distort the integration path in the complex $k$ plane by going around the point $k=0$ from below. Next, the integral in Eq. (2.21) decomposes into four 
contributions with integrands headed by $e^{-i k\left(r-\sigma_{i j}\right)}, e^{-i k\left(r+\sigma_{i j}\right)}$, $e^{-i k\left[r+\left(\sigma_{j}-\sigma_{i}\right) / 2\right]}$, and $e^{-i k\left[r+\left(\sigma_{i}-\sigma_{j}\right) / 2\right]}$, respectively, with each integrand having a single pole at $k=0$ of order $2 d-1$. If $0<r<\sigma_{i j}$, the first integral must be closed with an upper half circle of infinite radius, and the residue theorem yields a nonzero value. If $0<r<\left|\sigma_{j}-\sigma_{i}\right| / 2$, an additional nonzero contribution results from the third or fourth integral, depending on whether $\sigma_{i}>\sigma_{j}$ or $\sigma_{j}>\sigma_{i}$, respectively. On the other hand, if $r>\sigma_{i j}$, we must close the path with a lower half circle. As a consequence, the four contributions vanish, and so the RFA method yields Eq. (2.12).

This completes the proof on the equivalence between the PY and RFA solutions for binary mixtures of hard hyperspheres at odd dimensional space.

\section{CONCLUDING REMARKS}

PY and RFA theories are, in principle, alternative methods for calculating thermodynamic and structural functions of HS systems. The PY theory consists of the OZ relation (2.1) supplemented with the hard-core condition (2.5) and the genuine PY closure (2.12). In the RFA approach, however, one proposes a specific form, Eqs. (2.13)-(2.16), for the $s$ dependence of the Laplace functional $G_{i j}(s)$ defined by Eq. (2.3). This specific form includes $n+2=(d+1) / 2$ coefficients, $L_{i j}^{(m)}$, which are determined, in consistency with Eq. (2.7), by requiring the independent term in the Taylor series expansion of $s^{2} G_{i j}(s)$ to be $(d-2)$ !! and all the coefficients of $s^{2 q+1}$ with $q=0, \ldots, n$ to vanish.

In this paper we have shown by a direct verification that both methods are fully equivalent for binary mixtures of additive hard hyperspheres at odd dimensions. The proof is based on the analysis of the Fourier transform $\widehat{c}_{i j}(k)$ of the direct correlation function $c_{i j}(r)$ and proceeds along two main stages. In the first stage, use of Eqs. (2.13)-(2.16) has allowed us to derive Eq. (3.17), where $\widehat{\mathcal{P}}_{i j}(s), \widehat{\mathcal{Q}}_{i j}(s)$, and $\mathcal{D}(s)$ are polynomials. This result applies regardless of the values of the $n+2$ coefficients $L_{i j}^{(m)}$. In the second stage, we have proved that enforcement of Eq. (2.7) implies Eqs. (4.2), (4.7), and (4.8), so that Eq. (3.17) simplifies further to Eq. (4.6). Application of the residue theorem then yields Eq. (2.12), which completes the proof. An interesting feature of the proof is that the explicit expressions for the coefficients $L_{i j}^{(m)}$ as functions of the physical parameters of the fluid (density, mole fractions, and particle diameters) are not needed.

Although the proof presented in this paper has been restricted to the binary case $(\mathcal{N}=2)$, we conjecture that the structure of Eq. (4.6), and hence the validity of Eq. (2.12), remains valid for any number $\mathcal{N}$ of components in the framework of the RFA.

It is worth mentioning that, as done for three-dimensional mixtures [10] and for $d$-dimensional one-component systems [8], the RFA scheme can be extended beyond the PY level by adding extra terms $L_{i j}^{(n+2)}$ in Eqs. (2.14) and (2.16) and replacing $\delta_{i j}$ by $(1+u s) \delta_{i j}$ in Eq. (2.15). The $\mathcal{N}^{2}+1$ free parameters $L_{i j}^{(n+2)}$ and $u$ can be fixed by imposing given expressions for the contact values $g_{i j}\left(\sigma_{i j}^{+}\right)$and the thermodynamically consistent isothermal compressibility.

\section{ACKNOWLEDGMENTS}

The work of R.D.R. has been supported by the Consejo Nacional de Investigaciones Científicas y Técnicas (CONICET, Argentina) through Grant No. PIP 112-200801-01474. A.S. acknowledges support from the Ministerio de Ciencia e Innovación (Spain) through Grant No. FIS2010-16587 and the Junta de Extremadura (Spain) through Grant No. GR10158, partially financed by Fondo Europeo de Desarrollo Regional (FEDER) funds.
[1] J.-P. Hansen and I. R. McDonald, Theory of Simple Liquids (Academic, London, 2006).

[2] L. S. Ornstein and F. Zernike, Proc. Acad. Sci. Amsterdam 17, 793 (1914).

[3] J. K. Percus and G. J. Yevick, Phys. Rev. 110, 1 (1958).

[4] Theory and Simulation of Hard-Sphere Fluids and Related Systems, Lecture Notes in Physics, Vol. 753, edited by A. Mulero (Springer, Berlin, 2008).

[5] M. S. Wertheim, Phys. Rev. Lett. 10, 321 (1963).
[6] E. Thiele, J. Chem. Phys. 39, 474 (1963).

[7] J. L. Lebowitz, Phys. Rev. A 133, 895 (1964).

[8] R. D. Rohrmann and A. Santos, Phys. Rev. E 76, 051202 (2007).

[9] R. D. Rohrmann and A. Santos, Phys. Rev. E 83, 011201 (2011).

[10] S. B. Yuste, A. Santos, and M. López de Haro, J. Chem. Phys. 108, 3683 (1998).

[11] For convenience, the matrix $\mathrm{B}(s)$ introduced here differs from that in Ref. [9] by a multiplicative factor $s^{d}$. 